\title{
CO OBSERVATIONS OF ARP'S INTERACTING GALAXIES
}

\author{
YOSHIAKI SOFUE ${ }^{1}$, KEN-ICHI WAKAMATSU $^{2}$, YOSHIAKI TANIGUCHI $^{1}$, \\ NAOMASA NAKAI ${ }^{3}$, TOSHIHIRO HANDA ${ }^{1}$ \\ 1 Institute of Astronomy, University of Tokyo, Mitaka 181, Japan \\ 2 Physics Department, Gifu University, Gifu 505-11, Japan \\ 3 Nobeyama Radio Observatory, Nagano 384-13, Japan
}

\section{Starburst galaxies are interacting}

A number of starburst galaxies are included in the Atlas of Peculiar Galaxies (Arp 1966) which is the collection of peculiar-morphology galaxies showing mutual tidal interaction. A scenario of starburst can be summarized as follows (e.g. Sofue 1987, Noguchi 1988). Tidal interaction between galaxies causes an oval disturbance in the gravitational potential of a galaxy. The oval disturbance causes a bar in the innermost region of the galaxy. By this bar a galactic shock wave is excited, which results in rapid accretion of gas toward the central region. The accreted gas produces a dense ring or a core of molecular gas in the central region, from which massive stars are born. Intense UV radiation from the stars heats dust in the dense molecular gas core/ring, and the dust emits strong FIR emission.

\section{Does every interacting galaxy show starburst?}

This scenario seems to have been widely accepted. However, it has been based on the fact that the observed starburst galaxies or FIR luminous galaxies are often identified with interacting galaxies. Namely, the scenario is sample-dependent, where only active star-forming galaxies are taken into account. On the other hand, no systematic study of the behaviour of molecular gas in interacting galaxies, namely dynamics, distribution, and physical condition of the gas, has been made as yet. Some questions arise: is starburst inevitable as a consequence of galaxy-galaxy interaction? If so, in which phase of interaction does a starburst occur? What dynamics, distribution and physical condition does the molecular gas show during interactions? In particular, is the star-formation rate in the central regions higher in interacting systems?

To answer these questions, in particular the last one, we have conducted a ${ }^{12} \mathrm{CO}(J=1-0)$ line survey of interacting systems in the Arp's atlas. We have obtained spectra for the central regions of 43 galaxies, while no maps have been obtained. Observations were made in the winters of 1989 and 1990, using the 45-m telescope of the Nobeyama Radio Observatory with a HPBW of 17". The total observing time per data point (usually per galaxy) was 1-2 hours, so that the onsource integration time was about $20-40 \mathrm{~min}$. The rms noise of the resultant spectra of $10 \mathrm{~km} \mathrm{~s}^{-1}$ velocity resolution was about $\Delta T_{\mathrm{mb}} \simeq 10 \mathrm{mK}$. 


\section{Results and discussion}

Figure 1 shows a plot of far-IR luminosities $v$. CO luminosities for observed galaxies (filled circles). In the figure some known starburst galaxies ( $x$ ) and normal galaxies $(+)$ are plotted for comparison. Note that the CO luminosities of Arp galaxies are lower-limit values, because the telescope beam covered only the central few kpc regions. Even though we take this fact into account, we can see a tendency for the Arp galaxies to lie far above the line indicated by normal galaxies. From this we can see that the star-formation efficiency (FIR luminosity divided by molecular gas mass) is larger, and is comparable to that for starburst galaxies.

However, the sample number is too small to categorize the plots by interacting phases and type, for which we need more samples. We also need mapping to discuss gas dynamics during the interactions.

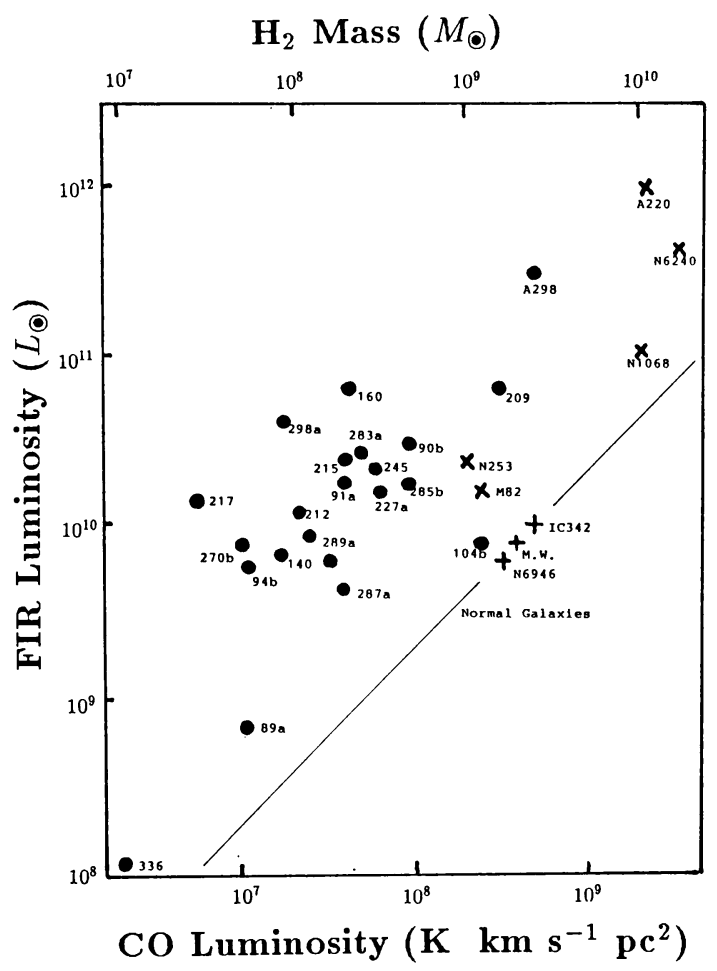

Figure 1 Plot of $L_{\mathrm{FIR}}$ versus $L_{\mathrm{CO}}$ for the detected Arp galaxies (•), starburst (x) and normal galaxies $(+)$.

\section{References}

Arp, H. (1966), Atlas of Peculiar Galaxies. (Calif. Inst. Tech.) p.48.

Noguchi, M. (1988), Astron. Astrophys. 203, 259.

Sofue, Y. (1987), Galactic and Extragalactic Star Formation, R. Pudritz (ed.). (Reidel:

Dordrecht), p. 409. 